\title{
Average Life Expectancy as a Criterion for Regional Risk Management
}

\author{
Sviatoslav Timashev ${ }^{1,2}$ \\ 1.Civil Engineering Institute, Ural State Federal University, Studencheskaya 54-A, Yekaterinburg, 620049, Russia \\ 2.Science \& Engineering Centre, Ural Branch, Russian Academy of Sciences, Studencheskaya 54-A, Yekaterinburg, \\ 620049, Russia \\ E-mail: timashevs@gmail.com
}

Received 27 June 2013

Accepted 5 February 2014

\begin{abstract}
The paper considers an important and complex interdisciplinary integration problem - providing, supporting and managing safety of interdependent critical infrastructures (ICIs) of a municipal area or region, embedded in the context and realities of modern society of risk. The problem is solved using a new unified quantitative criterion for managing regional risk, namely, the regional average life expectance (RALE) criterion, combined with a new algorithm for assessing the value of regional risk, which involves mechanics of catastrophes, computer modeling and risk-analysis theory.
\end{abstract}

Keywords: Average life expectancy, regional risk management.

\section{Introduction}

Public safety and security is an important objective, but diminishing of any type of risk requires additional expenditures. The share of resources that is being devoted by the society for achieving its safety must be continuously evaluated, having in mind such needs of society, as clean air and water, healthy food, housing, health care, social security benefits, pensions, education, etc., which also improve the longevity and quality of life.

But among all lines of diminishing risk the most important is decreasing industrial risk which is generated by the so called critical infrastructures (CIs).

Critical infrastructure is any large distributed renewable multicomponent geotechnical man-machineenvironment system (which consists of many objects and groups of people, who operate these objects). The $\mathrm{CI}$ is designed for providing safety and wellbeing of the population and sustainable development of the region, and also for supporting effective operation of a potential dangerous object (PDO), or a whole industry.
This is because the CIs generate the basic regional domestic product (RDP) as they provide for the wellbeing of the society in the most straightforward way. All the above components of risk are secondary as related to the overall risk. Therefore it stands for reason that management of regional risk may be boiled down to management of risk of ICI systems located in the region.

CIs are systems with following intrinsic features: non-economical responsibility (human factor HF, environment); functional, structural and time-wise redundancy; geometrical, physical, statistical and economical non-linearity. Modern CIs have, as their indelible parts, monitoring and/or control sub-system(s); risk based diagnostic subsystem; risk based predictive maintenance subsystem of asset integrity and safety; security/defense subsystem; other. All these specifics should be consistently accounted for during the design, operation and risk analysis (RA) of PDOs and CIs. RA of operating CIs is carried out using the full group of scenarios (FGS) concept. ${ }^{1,2,3}$

The key element when considering regional risk is the assessment of the behavior of the network of ICIs 
under the influence of natural, technological and intentional hazards. The ICI networks can be considered as conduits and at the same time as intermediary between the natural environment and the resource demands of the society (population living in the region). The ICIs are also the principal source of technological hazards in the region. The ICIs are designed to meet standards of safety and well-being for the population and environmental sustainability of the region, and also for supporting effective operation of potentially dangerous facilities, or functions. A point failure anywhere in the ICI can be rapidly propagated through the region with broad impacts on the environment and the regional population. Unified criteria for the assessment of these distributed impacts are needed to effectively evaluate the benefits of risk reduction.

The main conceptual problem of assessing, monitoring, and managing risk of ICIs is defined by following factors: l) the dimension of the problem is very large (potentially, tens of thousands of interdependent parameters); 2) the parameters involved are from different sciences and branches of engineering; 3) the ICI resilience/risk cannot be adequately described without explicitly accounting for the human factor.

The problem of regional risk management consists of following two parts: assessing the full possible damage and all of its components; designing means and methods for reduction the potential consequences of an initial failure in the system of ICIs. Hence, assessment of the full possible damage and of all its main components is the first part of the problem. The second part of the problem is to design means and methods which would reduce the potential consequences of an initial failure in the ICI.

The problems described above can be solved only through an inter-and multidisciplinary approach, and by convoluting the plethora of the heterogeneous parameters, which define the operation of the ICI, into just a few integral parameters, which should be simple to understand and use. Hence, before solving the problem in consideration, it is necessary to introduce some unified measures of safety/risk, which account for the human factor (HF) in socially meaningful terms.

Currently, following unified measures (generalized parameters) of safety/risk are considered, which allow accounting for the HF: ICI resilience; regional life expectancy; life quality index; ICI entropy. In this paper only the RALE criteria are discussed. The ICI resilience,
RLQI and ICI entropy criteria are discussed in Refs. 1-3 and 4.

The RALE is a non-additive parameter and permits combining seamlessly the technological reliability and safety of the ICI, its components and elements with economical parameters of their operation and social aspects of sustainable development of the region of their location and, hence, with the generalized problem of regional risk management. From this standpoint the central problem of regional (ICIs) risk management becomes optimization of the distribution of the always limited resources to improve the overall safety of systems of ICIs, and via this, the regional safety.

The paper describes principles and methodology which lead to achieving this goal.

\section{Overview of Research in Quantifying RALE as a Function of Different Economic Parameters}

Overview and analysis of existing world literature on this topic shows that most research is dedicated to creating models which combine ALE/RALE with some economic features of the country/region (GDP/RDP per capita). The very first attempt on tackling this problem seems to be due to S.H. Preston, ${ }^{5}$ who in 1975 started the studies of the relation between mortality and the general level of economic development using the logistic curve.

After his seminal work other authors started creating, mostly for demographic, financial ${ }^{6}$ and insurance ${ }^{7}$ audiences, different types of stochastic mortality models using real life data gathered from different population cohorts of different countries and geographic regions. ${ }^{6-}$ ${ }^{19}$ Among them the Lee-Carter ${ }^{14}$ and P-spline regression models should be mentioned, created for assessing the pricing risks for large insurance companies. Other stochastic models used include time series, quantile regression, the Poisson Log-bilinear model for mortality forecasting, ${ }^{8}$ and the Bayesian probabilistic projections of life expectancy for all countries, ${ }^{19}$ as well as models for efficiency estimation, and techniques for censored and truncated data ${ }^{17}$ and for smoothing the initial data. ${ }^{12,16}$ The initial data needed to construct the forecast models was collected mostly from England, Wales and the USA. ${ }^{9-11,18}$ The root causes of mortality in these papers were not fully addressed.

Further analysis of the world literature on this issue shows that main efforts are applied to forecast the 
mortality as it depends on actuarial data per se, as well as on some mortality reduction factors not related to technogenic causes (weather ${ }^{15}$ etc.). There are just a few papers which are dedicated to establishing a quantitative relationship between the ALE/RALE and the economic components of regional economics into which the GDP/RDP could be parsed., ${ }^{1,21}$ It stands to reason therefore, to create a model which would explicitly combine the different expenditures related to regional risk reduction and mitigation which comprise the RDP, with the RALE. Description of such a model is given below.

\section{Existing Approaches to Regional Risk and Safety Analysis}

Currently two approaches exist to assessing regional risk and safety.

The first approach can be described as the "from top to bottom" (TTB) method. This approach largely ignores the small scale events and zeroes in on regional scale consequences. It uses the input-output (I-O) model) ${ }^{20}$ balance method of the Nobel Prize winner Leontieff, in trying to assess the overall regional losses due to the imbalance/disruption caused by interruption of the operations of businesses and supplies of the regional ICIs. Due to the mega scale of the approach it does not account for the local events which are the genuine triggers of the incident/catastrophe and also are responsible for their branching and/or bifurcations, and, ultimately, for the structure of the whole disaster scenario as it develops in time and space, and concentrates only on the perceived final consequences (including the human factor involved in the catastrophe) of (mega) regional scale.

The second approach, proposed by the author ${ }^{21,22}$ and described below can be termed as the method "from bottom up (BU)". This model builds the risk model using as its "bricks" corresponding results of solutions of problems that relate to elements of critical infrastructures, and, finally, systems of CIs. It is constructed using the true multidisciplinary blockmodule principle, which prescribes that the output of the first local problem automatically serves as the input for the second local problem and so forth, down to the solution of the last problem in the logical chain of interconnected problems, which yields the sought new knowledge, A formalistic description of this approach is given by following scheme:

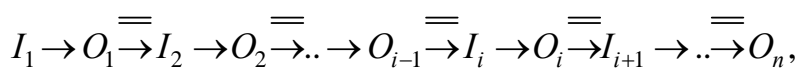

where $n$ is the total number of interconnected problems.

This multidisciplinary approach permits constructing the first approximation regional risk model, using as its components the corresponding already existing, off-theshelf engineering solutions which are related to fracture mechanics, integrity and reliability of PDOs, elements of CIs, and ICI systems.

These solutions can be found in the regulations and ruling documents institutionalized by a series of Federal laws of many countries, including Russia and the USA. According to these laws and documents, every entity which falls into the category of potentially dangerous object (PDO) is obliged to provide a declaration and a passport of its safety, and a risk map, which depicts the individual risk in the territory of the site and its surroundings. These documents contain a chapter which describes, in quantitative terms, the operational risk of this entity and a chapter that describes the means that are needed for mitigating the catastrophe (earth moving and other types of machines, transportation, materials, workforce, and financial means) for the worst case scenario and the average scenario. The risk (failure) analysis is conducted using a set of State / EMERCOM / FEMA approved recommended practices. EMERCOM is the Russian analog of FEMA.

Recommended practices are based on solutions of relevant problems of fracture mechanics, blast, fire, spill, filtration, water and air pollution, and descriptions of their consequences in typical scenario settings. They provide some guidelines as to how assess the number of fatalities and the monetary value of lost life or limb; prescribe how to assess the damage inflicted by a catastrophe and to present the collective risk specific for the PDO in consideration.

This approach is implemented, particularly, in Russia. ${ }^{1,2,3}$ Similar guidance in the US is provided by the Universal Task List and the Target Capability list that provide a hierarchy of missions, objectives, functions, and tasks that map to nominal regional capabilities that can be characterized in terms of resource requirements. ${ }^{6}$

\section{Quantitative Description of the Average Life Expectancy (ALE)}

The most valuable asset of any society are its people. The most valuable trait of a human being is her/his life. 
The most valuable parameter of a human life is its longevity in good health.

RALE at birth is a non additive (non linear) parameter which permits combining parameters of complex safety of elements, structures and ICI systems with economic parameters of the operation and social aspects of sustainable development of the region.

RALE provides seamless fusion of separate specific problems of safety/reliability of CIs and their elements with the generalized problem of regional risk management. It also has a biological «ceiling» and some properties of a fractal, is the solution of a system of differential equations, and has the form of a complex logistic curve, which is a function of time. It depends on the current value of ALE and on how optimal the society distributes the year-by-year the regional DP on accumulation of wealth, consumption, and on safety of the system of the ICIs, its employees, and the adjacent to it population from the possible influence of incidents of different origin (nature, technological, premeditated).

In the last component it is necessary to:

- Single out those means (shares of RDP and of the PDOs budget), which could be (are) allotted to mitigate disasters and catastrophes of the ICIs' components and, accordingly,

- Define, what would be the decrease/increase of the number of fatalities/injuries in the region in consideration due to natural/technogenic incidents,

- Assess how quantitatively this will influence the RALE,

- Balance the benefit from increasing safety (here increasing RALE), and the cost of decreasing risk.

The ability of any society to prevent premature death/injury of its people is finite and restricted by its capability to create societal wealth; hence the central problem of management of any risk (including technological risk) becomes optimization of the distribution (by volume and place of application) of the always limited resources to mitigate risk, using, particularly, the RALE criterion.

ALE is a convenient characteristic for assessing the quality of life, because it continues to make sense with the size of the society in consideration shrinking. Indeed, it is possible to calculate ALE for the nation/country as a whole, as well as for a separate region, industry, ICI, PDO and even for an individual.

In this research following formula (based on the method of ultimate, or final, expenditures) is used for evaluating the GDP: ${ }^{23}$

$$
c(t)+a(t)+d(t)+c_{\text {exp }}(t)=R D P(t)
$$

where: $c(t)$ is the final consumption, excluding state expenditures; $a(t)$ is the overall accumulation of wealth; $d(t)$ are the state expenditures; $c_{\text {exp }}(t)$ is the net export value (if import is larger than export, this component may be negative).

This paper is concentrating on the fact that by decreasing technological risk the state, region, municipality ALE is extended.

In the general case ALE at any arbitrary time $t$ depends on the main components of GDP/RDP/MDP:

$$
T_{a v}(t)=T_{a v}[c(t), a(t), d(t)]
$$

where $c(t)$ is the final consumption; $a(t)$ is the accumulation of wealth; $d(t)$ is the state expenditures on safety.

It should be noted that in the above functional equation the arguments (functions) are specific, being related to a statistically average resident of the region in consideration. The relationship (2) can be interpreted as a certain utility function of social development of the region. This function has to have following obvious properties: a biological growth limit $T_{u}>0$; with the growth of consumption by and protection of the regional residents from all types of risk the $T_{a v}$ has to monotonically increase; hence, $\partial T_{a v} / \partial c \geq 0$; $\partial T_{a v} / \partial d \geq 0$; if all other arguments are of fixed value, then $\partial T_{a v} / \partial a \leq 0$. This reflects the negative influence of increasing technogenic hazards (due to capitalization of those industries which generate the RDP) on the RALE.

It should be noted that the dependence of ALE on its arguments differs on different stages of the social development of the region in consideration. At low levels of consumption it feebly influences the ALE growth; but after reaching a certain level of consumption its influence on ALE growth becomes much larger - a much smaller (than in the initial stage) increment in consumption leads to significantly larger increment in ALE growth. In its third phase the ALE function starts saturating - its dependence from consumption again starts to fall into the same pattern as in the first phase (but at a much higher level). In this phase in order to increase the ALE by the same increment as in phase one, much more consumption is needed. This signals that the ALE starts reaching its ultimate level. Hence, at phase three $\partial^{2} T_{a v} / \partial c^{2} \leq 0$. 
The ALE function has the same character of dependency with respect to its other two arguments.

From all the above it flows out that the ALE function as a function of arguments the $c, d$ and $a$ has to have the form of a logistic curve. Taking into account the above restrictions on the arguments, following system of differential equations (SDE), can be obtained:

$$
\left\{\begin{array}{l}
\frac{\partial T_{a v}}{\partial c(t)}=\alpha_{c}(t) T_{a v}\left(T_{u}-T_{a v}\right) / T_{u}, \\
\frac{\partial T_{a v}}{\partial d(t) / a(t)}=\alpha_{d / a}(t) T_{a v}\left(T_{u}-T_{a v}\right) / T_{u},
\end{array}\right.
$$

Where parameters $\alpha_{c}, \alpha_{d / a}$ characterize the ALE growth rate and $T_{u}$ is the biological "ceiling" (in an "ideal environment"), without accounting for the external forces which may lower it down. Biologists studying the problem consider that the genetic content of human species permit human longevity up to 150 years. Currently, the documented and registered world record of human longevity is just over 122 years. ${ }^{22}$

It stands to reason, however, that this limit shouldn't be one across the board; it rather should be estimated as a quantile, assessed for each specific geographical region and type of environment, which accounts for the regional wealth, overall level of pollution, and cultural and eating habits.

Solution of the first Eq. (3) will be a logistic curve:

$$
T_{a v}[c(t)]=\frac{T_{u} T_{0} e^{\alpha_{c}(t) c(t)}}{T_{u}+T_{0}\left(e^{\alpha_{c}(t) c(t)}-1\right)}=\frac{T_{u}}{1+\left(\frac{T_{u}}{T_{0}}-1\right) e^{-\alpha_{c}(t) c(t)}}
$$

Solution for the second Eq. (3) is:

$$
\begin{gathered}
T_{a v}[d(t) / a(t)]=\frac{T_{u} T_{0} e^{\alpha_{d / a}(t) d(t) / a(t)}}{T_{u}+T_{0}\left(e^{\alpha_{d / a}(t) d(t) / a(t)}-1\right)}= \\
=\frac{T_{u}}{1+\left(\frac{T_{u}}{T_{0}}-1\right) e^{-\alpha_{d / a}(t) d(t) / a(t)}} .
\end{gathered}
$$

Where $T_{0}$ is the value of ALE at the initial time $t=0$, i.e. $T_{0}=T_{a v}(0)=T_{a v}[c(0)]=T_{a v}[d(0) / a(0)]$.

Then the general solution of the system of DE (3) will be:

$$
T_{a v}[a(t), c(t), d(t)]=\frac{T_{u}}{1+\beta_{1} e^{-\alpha_{c}(t) c(t)}+\beta_{2} e^{-\alpha_{d / a}(t) d(t) / a(t)}}
$$

Here parameters $\beta_{1}, \beta_{2}, \beta_{3}$ reflect the partial influences of $c(t), d(t), a(t)$ at initial time $t=0$. When $t=0, T_{a v}[a(0), c(0), d(0)]$ should be equal to $T_{0}$, therefore $\beta_{1}+\beta_{2}=\frac{T_{u}}{T_{0}}-1$. For instance, if the GDP components $c(t), d(t), a(t)$ at $t=0$ own $k_{c}, k_{d}, k_{a}$ shares of the RDP correspondingly, then the coefficients $\beta_{1}, \beta_{2}, \beta_{3}$ can be found from formulae:

$\beta_{1}=p k_{c}, \beta_{2}=p k_{d} / k_{a}, p=\frac{T_{u}-T_{0}}{T_{0}\left(k_{c}+k_{d} / k_{a}\right)}$,

where $p$ is a correcting coefficient.

Parameters $\alpha_{c}(t), \alpha_{d}(t), \alpha_{a}(t)$ can be interpreted as the rates of ALE growth and are a measure of the instantaneous and specific ALE growth rates. It is described as the number of years per unit of measurement of $c(t), d(t)$ or $a(t)$ per person. For instance, if the GDP per person is measured in thousands of monetary units (m.u.), then the parameter $\alpha_{c}(t)$ will be measured in units of [years/1000m.u.].

The coefficients $\alpha_{c}(t), \alpha_{d}(t), \alpha_{a}(t), \alpha_{d / a}(t)$ can be defined using following formulae:

$$
\begin{aligned}
& \alpha_{c}(t)=-\frac{\ln \left(\frac{T_{u}-T_{a v}(t)}{T_{a v}(t) Q}\right)}{c(t)}, \quad \alpha_{d}(t)=-\frac{\ln \left(\frac{T_{u}-T_{a v}(t)}{T_{a v}(t) Q}\right)}{d(t)}, \\
& \alpha_{a}(t)=-\frac{\ln \left(\frac{T_{u}-T_{a v}(t)}{T_{a v}(t) Q}\right)}{a(t)}, \quad \alpha_{d \prime a}(t)=\frac{\alpha_{a}(t)}{a_{d}(t)},
\end{aligned}
$$

where $T_{a v}(t), c(t), d(t), a(t)$ are the statistical data of past years, which are used to fine tune the model, and $Q=\left[\left(T_{u} / T_{0}\right)-1\right]$.

\section{RALE Optimization}

If the maximal growth of RALE is taken as the criterion of social development (sustainable growth): $\bar{T}_{a v} \rightarrow \max$ where $\bar{T}_{a v}=\int_{0}^{T} T_{a v}(t) d t / T$ is the life expectancy of an statistically average individual, obtained by averaging over the forecast time interval $T$, then the problem of optimizing RALE comes down to the problem of unconditional optimization of a multi-variable function: 


$$
\int_{0}^{T_{u}} T_{a v}(a(t), c(t), d(t)) d t / T_{u} \rightarrow \max ,
$$

where $\left[0, T_{u}\right]$ is the forecasting interval depth.

This problem is solved using the Nelder-Mead algorithm (a.k.a. as the simplex method) which performs a unconditional optimization of a multi argument function, does not use the gradient functions and, therefore, is applicable to non-smooth functions and/or functions with noise. ${ }^{24}$

\section{Prediction of Rale}

Forecasting of the RALE was conducted using available statistical data about the US and Russia GDP and their components:

- $\quad$ for the USA - according to the Bureau of Economic Analysis years 2000-2010;

- for Russia, Sverdlovsk Region and the city of Yekaterinburg - according to the data provided by RosGosStat, years 2000-2009.

In Tables 1-4 are presented for comparison the actual values of ALE and their assessments using the logistic model developed in this paper.

\section{Assessments of Regional, Collective and Societal Risk}

In Figures 1-4 the forecasted values of RALE are presented, depending on different values of $T_{u}$.

It can be observed that the proposed model for predicting RALE gives very accurate assessments of the real life data. This permits using the model for predicting the future values of RALE under different assumptions about the RDP growth and its distribution between the three components $[c(t), d(t), a(t)]$.

Figures 1-4 show that the "ceiling" parameter $T_{u}$ is not of great importance, as it does not significantly change the outcome. For instance, the difference of RALE calculated for $T_{u}=80$ yrs and $T_{u}=120 \mathrm{yrs}$ is around 1.5\% (see Fig. 2, year 2015).

All calculations related to the RALE assessments and visualization of the results were conducted by A.V. Bushinskaya, $\mathrm{PhD}$.

Table 1. Comparison table of actual values of RALE for men in USA and their assessments (years) at $T_{u}=85$ years

\begin{tabular}{lccccccccccc}
\hline \multicolumn{1}{c}{ Years } & 2000 & 2001 & 2002 & 2003 & 2004 & 2005 & 2006 & 2007 & 2008 & 2009 & 2010 \\
\hline Actual Values & 74.10 & 74.20 & 74.30 & 74.50 & 74.90 & 74.90 & 75.10 & 75.40 & 75.50 & 75.70 & 75.90 \\
$\begin{array}{l}\text { Assessments } \\
\text { Assessments - Actual }\end{array}$ & 74.14 & 74.27 & 74.41 & 74.57 & 74.73 & 74.91 & 75.09 & 75.29 & 75.49 & 75.70 & 75.91 \\
Values & -0.04 & -0.07 & $\mathbf{- 0 . 1 1}$ & -0.07 & 0.17 & -0.01 & 0.01 & $\mathbf{0 . 1 1}$ & 0.01 & 0.00 & -0.01 \\
\hline
\end{tabular}

Table 2. Comparison table of actual values of RALE for the population of Russia and their assessments (years) at $\mathrm{T}_{\mathrm{u}}=80$ years

\begin{tabular}{lcccccccccc}
\hline \multicolumn{1}{c}{ Years } & 2000 & 2001 & 2002 & 2003 & 2004 & 2005 & 2006 & 2007 & 2008 & 2009 \\
\hline Actual Values & 65.34 & 65.23 & 64.95 & 64.85 & 65.27 & 65.30 & 66.60 & 67.51 & 67.88 & 68.67 \\
Assessments & 64.75 & 64.87 & 65.14 & 65.47 & 65.84 & 66.24 & 66.68 & 67.14 & 67.63 & 68.14 \\
Assessments - Actual Values & $\mathbf{0 . 5 9}$ & 0.36 & -0.19 & -0.62 & -0.57 & $\mathbf{- 0 . 9 4}$ & -0.08 & 0.37 & 0.25 & 0.53 \\
\hline
\end{tabular}

Table 3. Comparison table of actual values of RALE for men in Sverdlovsk region and their assessments(years) at $T_{u}=70$ years

\begin{tabular}{lcccccccccc}
\hline \multicolumn{1}{c}{ Years } & 2000 & 2001 & 2002 & 2003 & 2004 & 2005 & 2006 & 2007 & 2008 & 2009 \\
\hline Actual Values & 57.99 & 57.42 & 57.28 & 57.80 & 57.88 & 60.15 & 61.19 & 61.47 & 62.27 & 57.99 \\
Assessments & 56.73 & 57.48 & 58.12 & 58.74 & 59.37 & 60.00 & 60.64 & 61.27 & 61.88 & 56.73 \\
Assessments - Actual Values & $\mathbf{1 . 2 6}$ & -0.06 & -0.84 & $\mathbf{- 0 . 9 4}$ & -1.49 & 0.15 & 0.55 & 0.20 & 0.39 & $\mathbf{1 . 2 6}$ \\
\hline
\end{tabular}

Table 4. Comparison table of actual values of RALE for women in Yekaterinburg and their assessments (years) at $\mathrm{T}_{\mathrm{u}}=80$ years

\begin{tabular}{lccccc}
\hline \multicolumn{1}{c}{ Years } & 2005 & 2006 & 2007 & 2008 & 2009 \\
\hline Actual Values & 2005 & 2006 & 2007 & 2008 & 2009 \\
Assessments & 72.91 & 74.05 & 74.97 & 75.20 & 75.56 \\
Assessments - Actual Values & 73.39 & 73.98 & 74.55 & 75.10 & 75.63 \\
\hline
\end{tabular}




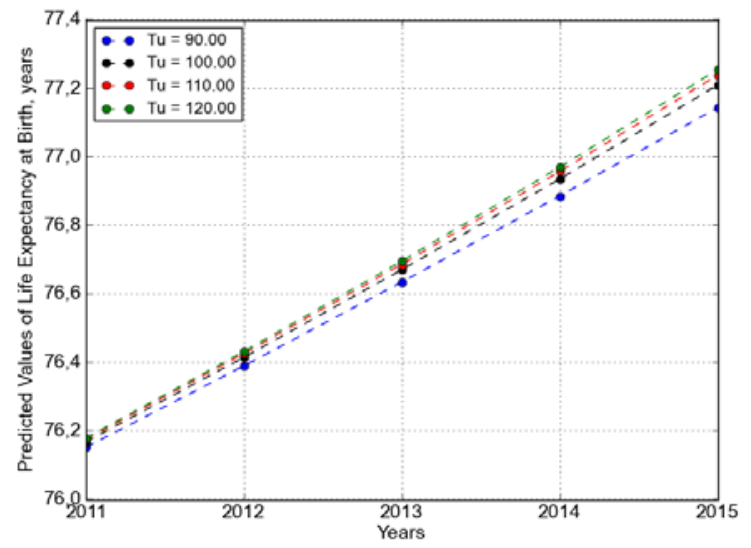

Fig. 1. Predicted ALE for USA (men).

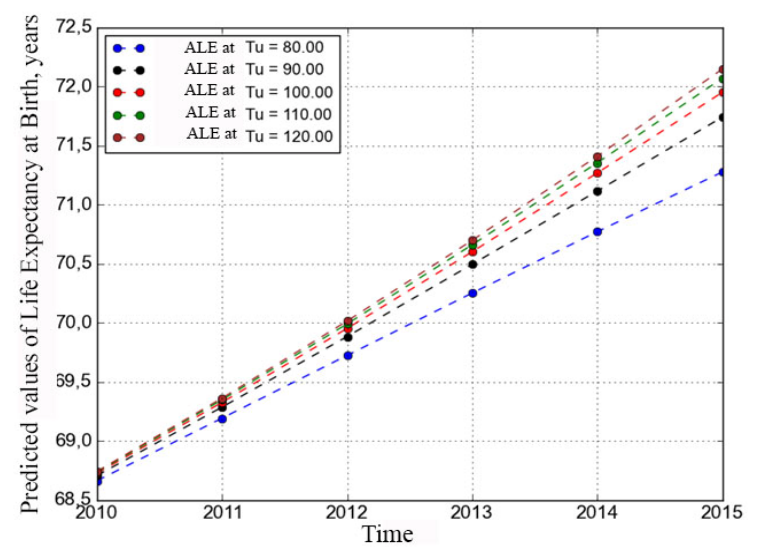

Fig. 2. Predicted ALE for Russia.

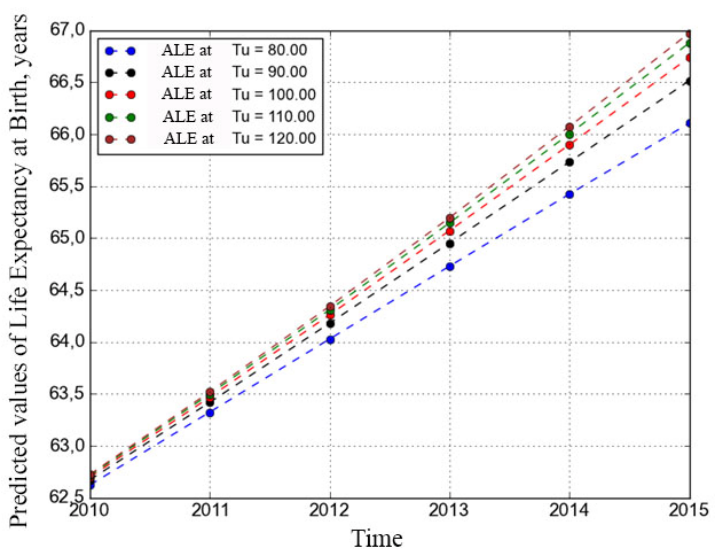

Fig. 3. Predicted ALE for Sverdlovsk region (men).

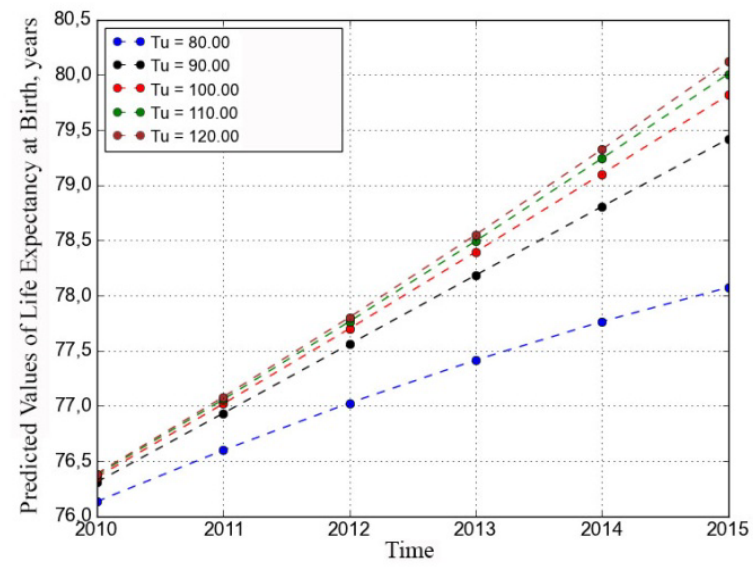

Fig. 4. Predicted ALE for Yekaterinburg (women).

\section{Assessments of Regional, Collective and Societal Risk}

The formulas given above permit assessing the influence of human losses during catastrophes of different nature on the decrease of the RALE.

Medical entities/organizations of different rank-from world-wide to local polyclinics and hospitals- possess statistics which permit assessing the statistical average time loss due to different types of illnesses. Generalizing this data over a region which is involved in a particular incident or catastrophe, it is possible to assess the total time loss of life - years by the residents of the region due to an incident. ${ }^{1,2,3}$

Distribution of risks over a region due to technogenic incidents and natural catastrophes can be described as a product of two independent probability density functions (PDFs). One of them describes the probabilistic distribution of the destruction factor(s) over the destruction zone $\Omega$ (DZ). The other describes the distribution of the employees over the territory of the potentially dangerous object (PDO) as well as of the citizens who live adjacent to the same territory. Hence, it is possible to write that the individual risk IR for a $k$ th type of incident (catastrophe)

$$
{ }_{k} \operatorname{IR}\left(x_{i}, y_{j}\right)=\sum_{i} \sum_{j} \Pi\left(x_{i}, y_{j}\right) \cdot P_{k} \cdot P_{\bar{q}, k} \cdot P_{m, k}
$$

where: $x_{i}, y_{j}$ is a point which belongs to the DZ: $\left(x_{i}, y_{j}\right) \in \Omega ; \operatorname{IR}\left(x_{i}, y_{j}\right)$ is the probability of death of an individual being in the geographical point $x_{i}, y_{j} ; P_{k}$ is 
the probability of a $k$-th type incident; $P_{\bar{q}, k}$ is the probability of influence by the vector of destruction factors of size $\bar{q}$ during a $k$-th type incident; $P_{m, k}$ is the probability of a mortality/ loss of limb in the case when an individual is influenced by the vector of destruction factors of size $\bar{q}$.

Parameters which enter Eq. (4) are defined in the following way: The probabilities $\Pi\left(x_{i}, y_{j}\right)$ are accessed via the statistical data (averaged over 24 hour intervals) of people being in DZ and occupying locations with coordinates $\left(x_{i}, y_{j}\right) \in \Omega ;$ the probabilities $P_{m, k}$ are calculated using corresponding probit functions; values of $P_{\bar{q}, k}\left(x_{i}, y_{j}\right)$ are defined on the basis of laws of physics and chemistry as related to the distribution of the destruction factors in the DZ; finally, the $P_{k}$ are found using corresponding statistical data.

In management of risk it is crucial to account for the scale of the incident/catastrophe, which involves deaths of groups of people due to an unfortunate combination (a vector) of destructive factors. This can be achieved by calculating the societal risk (SR).

In the risk analysis theory SR is defined as the mathematical expectation of lethalities due to a technogenic catastrophe. At this the probability of the same incident is explicitly accounted for.

Collective risk (CR) is a specific case of social risk (SR) during an incident at the PDO. Usually it is related to employees of the PDO and to people who live adjacent to it.

Assessment of societal and collective risk can be achieved using following formula:

$$
\begin{aligned}
{ }_{k} \mathrm{SR}(\mathrm{CR})=N \iint_{(x, y) \in \Omega} P_{k} \cdot P_{\bar{q}, k}\left(x_{i}, y_{j}\right) . \\
\cdot P_{m, k}\left(x_{i}, y_{j}\right) \Pi\left(x_{i}, y_{j}\right) d x d y .
\end{aligned}
$$

If all possible hazards are statistically independent, then $R_{\Sigma}=\sum_{i} R_{i}$, where $R_{\Sigma}$ is the integral risk; $R_{i}$ are the partial risks.

In many cases risks are mutually dependent and, moreover, some types of risks (i.e., fires) "consume" risks due to the initiation of the incident (structure collapse, explosions etc.).

In this case the integral risk is assessed as the union (conjunction) of all possible risks: $R_{\Sigma}=\cup_{i} R_{i}$. Taking into account the possible number of people involved in each threat/ accident and summing up the total regional risk, the value of the RALE decrease is assessed. This value is then used as a restriction or as the goal when solving corresponding optimization problems, which are formulated below.

\section{Selecting Risk Mitigating Means}

\subsection{Direct problem}

With given means for improving safety $S_{\text {giv }}$ choose such a set of measures that maximizes reduction of incident probability $Q_{i}=P(A)$.

$$
\left\{\begin{array}{l}
\sum_{i} \Delta Q_{i} \rightarrow \max \\
\sum_{i} S_{i} \leq S_{g i v}
\end{array}\right.
$$

\subsection{Inverse problem}

With minimal expenditures $\sum_{i} S_{i}$ choose such a set of measures, implementation of which lowers the incident probability $Q_{i}=P(A)$ down to an acceptable (assigned) level $P_{a c c}(A)$ :

\section{Real Life Examples}

As a partial illustration of the described above approach some results are given below.

In Figure 5 the risk map of a district of the city of Yekaterinburg is shown, which gives an idea of how the individual risk is distributed on the territory in consideration. This document is being used by the City Administration for making decisions, related to providing safety of the citizens, lands and its infrastructure. $^{25}$

The second example (Fig. 6) is related to a PDO, namely, the jet fuel line which provides the Manchester (UK) Airport with airplane fuel. The IR map for the kerosene pipeline ROW permits assessing risk for the population which lives in its vicinity.

By integrating the total risk over the territory, the decrease in RALE can be assessed, and then compared to the actual level. If the level is too high, then the inverse optimization problem from 8.2 has to be solved. 


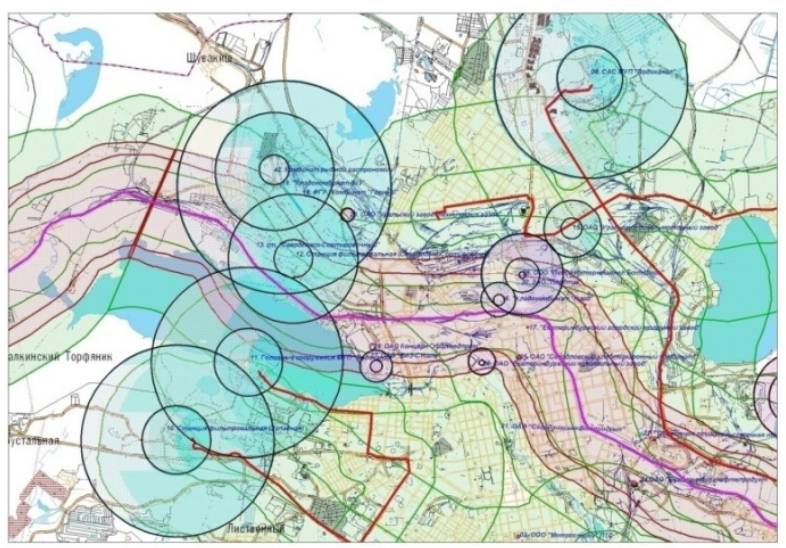

Fig.5. The Individual risk map for the city of Yekaterinburg.

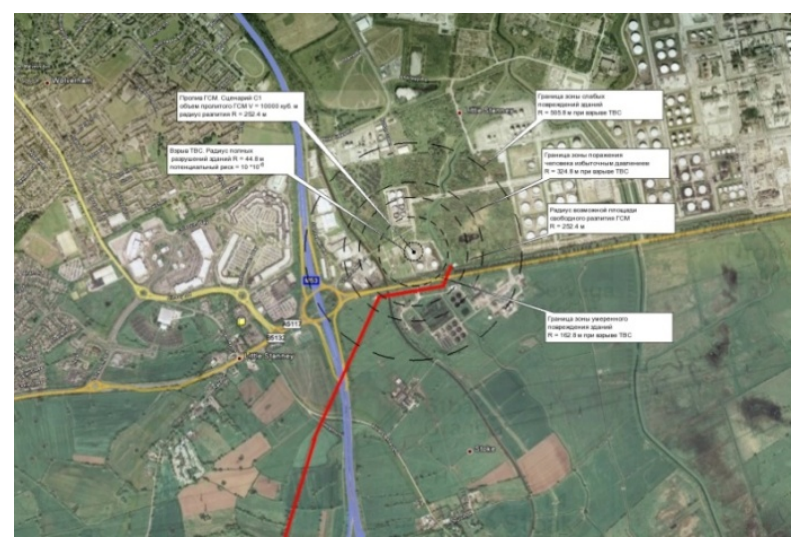

Fig. 6. Potential risk for the Manchester jet fuel pipeline. Boundaries of high risk area for a rupture of a kerosene tank $\mathrm{V}$ $=10000 \mathrm{~m}^{3}$ followed by a fuel air mixture explosion.

\section{Conclusion}

This present study gives the following conclusions:

(1) The RALE criterion is proposed as an effective means for convoluting a multidimensional heterogeneous problem of regional risk assessment and mitigation into a single dimension problem.

(2) The proposed FBU method of regional risk assessment permits utilization of the true multidisciplinary approach to problem solving, is repeatable and based on real data statistics.

(3) Cases of predicting the RALE for the USA, Russia, Sverdlovsk Region and the city of Yekaterinburg are presented, as a whole and separately, for women and men, by decreasing of the operating risk of potentially dangerous objects and ICI systems.

\section{References}

1. S. A. Timashev, Management of territorial critical infrastructures using the life quality index, in Proc. 1st All Russia Conf. Safety of Critical Infrastructures and Territories (Ural Branch Russian Academy of Sciences, Yekaterinburg, 2007) (in Russian).

2. S. A. Timashev, Management of risk of interdependent systems of critical infrastructures and territories, in Proc. II All Russia Conf. Safety of Critical Infrastructures and Territories (Ural Branch Russian Academy of Sciences, Yekaterinburg, 2008), ISBN 5-7691-2016-9, pp. 7-9 (in Russian).

3. S. A. Timashev and A. N. Tyrsin, Entropy approach to risk-analysis of critical infrastructures systems, in Proc. 1st Int. Conf. Vulnerability and Risk Analysis and Management (ICVRAM 2011) and 5th Int. Symp. Uncertainty Modeling and Analysis (ISUMA 2011), pp. 147-154.

4. J. S. Nathwany, N. C. Lind and M. D. Pandey, Affordable Safety by Choice: the Life Quality Method (Inst. for Risk Research, University of Waterloo, Waterloo, 1997).

5. S. H. Preston, The changing relation between mortality and level of economic development, Population Studies 29(2) (1975) 231-248. Reprinted in the Inter. J. of Epidemiology 36 (2007) 484-490.

6. D. T. Breeden, An intertemporal asset pricing model with stochastic consumption and investment opportunities, $J$. of Financial Economics 7(3) (1979) 265-296.

7. P. Barrieu and L. Albertini, The Handbook of InsuranceLinked Securities (Wiley Finance, 2009).

8. N. Brouhns, M. Denuit and I. van Keilegom, Bootstrapping the Poisson log-bilinear model for mortality forecasting, Scandinavian Actuarial J. (3) (2005) 212-224.

9. A. J. G. Cairns, D. Blake and K. Dowd, A two-factor model for stochastic mortality with parameter uncertainty: theory and calibration, $J$. of Risk \& Insurance 73(4) (2006) 687-718.

10. A. J. G. Cairns, D. Blake and K. Dowd, Modeling and management of mortality risk: a review, Scandinavian Actuarial J. (2) (2008) 79-113.

11. A. J. G. Cairns, D. Blake, K. Dowd, G. D. Coughlan, D. Epstein, A. Ong and I. Balevich, A quantitative comparison of stochastic mortality models using data from England \& Wales and the United States, North American Actuarial J. 13(1) (2009) 1-35.

12. I. D. Currie, M. Durban and P. H. C. Eilers, Smoothing and forecasting mortality rates, Statistical Modeling 4(4) (2004) 279-298.

13. N. Fournier and S. Meulleard, A microscopic probabilistic description of a locally regulated population and macroscopic approximations, Annals of Applied Probability 14(4) (2004) 1880-1919.

14. S. Haberman and E. Renshaw, A cohort-based extension to the Lee-Carter model for mortality reduction factors, 
Insurance, Mathematics, Statistics and Economics 38(3) (2006) 556-570.

15. S. Jewson and A. Brix, Weather Derivative Valuation: the Meteorological, Statistical, Financial and Mathematical Foundations (Cambridge University Press, 2005).

16. J. Kirkby and I. Currie, Smooth models of mortality with period shocks, Statistical Modelling 10(2) (2010) 177196.

17. J. P. Klein and M. L. Moeschberger, Survival Analysis: Techniques for Censored and Truncated Data (SpringerVerlag, New York, 2003).

18. R. D. Lee and L. Carter, Modeling and forecasting the time series of US mortality, J. of the American Statistical Association 87(419) (1992) 659-671.

19. J. L. Chunn, A. E. Raftery and P. Gerland, Bayesian probabilistic projections of life expectancy for all countries, Working Paper no. 105 (Center for Statistics and the Social Sciences University of Washington, Oct. 28, 2010).

20. Y. Y. Haimes, Risk Modeling, Assessment and Managemen (Wiley \& Sons, NY, 1998), 872 p.
21. S. A. Timashev, Resilience and preparedness of critical infrastructures, in Proc. 1st Int. Conf. Vulnerability and Risk Analysis and Management (ICVRAM 2011) and 5th Int. Symp. Uncertainty Modeling and Analysis (ISUMA 2011), pp. 764-771.

22. S. A. Timashev and V. N. Alekhin, Monitoring of Critical Infrastructures and Territories as the Base for Predictive Maintenance and Providing Regional Safety, 2013, Far East Branch, Russian academy of Sciences.(in Russian.)

23. N. P. Tikhomirov, I. M. Potravny and T. M. Tikhomirova , Methods of Ecological-Economical RiskManagement. Manual (Unity, Moscow, Russia, 2003), 349 p. (in Russian).

24. J. A. Nelder and R. Mead, A simplex method for function minimization, J. Computer 7 (1965) 308-313.

25. E. S. Guryev, L. V. Poluyan and S. A. Timashev, Methodology of constructing dynamic risk maps for large metropolitan areas, in Proc. 1st Int. Conf. Vulnerability and Risk Analysis and Management (ICVRAM 2011) and 5th Int. Symp. Uncertainty Modeling and Analysis (ISUMA 2011), pp. 716-723. 